\title{
MARCH: factors associated with weight loss in patients with newly diagnosed type 2 diabetes treated with acarbose or metformin
}

\author{
Na Wang, Jin-Ping Zhang, Xiao-Yan Xing, Zhao-Jun Yang, Bo Zhang, Xin Wang, Wen-Ying Yang
}

Department of Endocrinology, China-Japan Friendship Hospital, Beijing, China

Submitted: 26 August 2016

Accepted: 15 April 2017

Arch Med Sci 2019; 15, 2: 309-320

DOI: https://doi.org/10.5114/aoms.2018.75255

Copyright @ 2018 Termedia \& Banach

\begin{abstract}
Introduction: In this secondary analysis of the Metformin and AcaRbose in Chinese as the initial Hypoglycaemic treatment (MARCH) trial, we evaluated what demographic and clinical factors were associated with reduction in weight. We also assessed the effects of acarbose and metformin treatment on weight reduction.

Material and methods: We analyzed the demographic and clinical laboratory values from the 784 patients with type 2 diabetes of the MARCH study who were treated for 48 weeks with acarbose or metformin. We determined the association of the different parameters with a weight reduction of $\geq 2 \mathrm{~kg}$ in patients using univariate and multivariate analysis.

Results: In patients treated with acarbose, males were less likely than females to lose $\geq 2 \mathrm{~kg}$ of weight $(p=0.025)$. Higher baseline $\mathrm{HbA}_{11}$ levels and lower decreases from baseline in fasting plasma glucose (FPG) levels after 48 weeks of treatment were negatively associated with losing $\geq 2 \mathrm{~kg}$ of weight (both, $p<0.05$ ). Higher baseline glucagon AUC was also positively associated with reducing weight by $\geq 2 \mathrm{~kg}(p=0.010)$. In patients treated with metformin, change from baseline in whole body insulin sensitivity increased the odds of having a weight reduction of $\geq 2 \mathrm{~kg}(p=0.014)$.

Conclusions: This study found that for both acarbose and metformin, control of FPG significantly impacted weight loss. Baseline AUC for glucagon in patients treated with acarbose and an increase of whole body insulin sensitivity after 48 weeks of treatment with metformin were important factors for weight reduction.
\end{abstract}

Key words: weight loss, type 2 diabetes, metformin, acarbose, MARCH trial.

\author{
Corresponding author: \\ Dr. Na Wang \\ Department of \\ Endocrinology \\ China-Japan \\ Friendship Hospital \\ 2 Yinghua East Road \\ Chaoyang District \\ Beijing, 100029, China \\ Phone: +8601084205716 \\ Fax: +8601084205664 \\ E-mail: ywying_1010@163.com
}

\section{Introduction}

Being overweight or obese can increase the risk of cardiovascular disease in patients with type 2 diabetes. Achieving good weight control is a critical component of managing diabetes [1,2]. This is of particular concern given that many patients with type 2 diabetes are obese and that some types of antidiabetic agents (insulin, thiazolidinediones, and sulfonylurea) have the unwanted side effect of promoting weight gain [1]. In addition, a significant percentage of Chinese patients with newly diagnosed type 2 diabetes are overweight [1].

Several drugs, but not all, used to treat and manage type 2 diabetes promote weight gain. Metformin and acarbose, an $\alpha$-glucosidase inhibitor, are 
used to treat type 2 diabetes and are not associated with weight gain and also decrease the risk of cardiovascular disease [3, 4]. Acarbose slows carbohydrate digestion and inhibits postprandial increases in plasma glucose levels [4]. Evidence suggests that stimulation of the fasting and postprandial levels of the glucagon-like peptide (GLP-1) by acarbose and metformin may impact the benefit of these drugs on weight and other clinical parameters $[5,6]$.

The Metformin and AcaRbose in Chinese as the initial Hypoglycaemic treatment $(\mathrm{MARCH})$ study was a 48-week, randomized, controlled, non-inferiority, multi-center study that included 788 patients newly diagnosed with type 2 diabetes [7]. MARCH evaluated the effectiveness of acarbose and metformin in treating Chinese patients. Both treatments can reduce bodyweight, and acarbose treatment resulted in more weight reduction than metformin treatment at weeks 24 and 48 [7]. In this secondary analysis, we used univariate and multivariate analysis to evaluate what demographic and clinical characteristics impact weight reduction in patients from the MARCH study who were treated with acarbose or metformin.

\section{Material and methods}

\section{Study participants and study design}

The MARCH trial (Chinese Clinical Trial Registry number: ChiCTR-TRC-08000231) was performed in accordance with the Declaration of Helsinki, and was approved by the appropriate ethic committees of the different Chinese clinical sites [7]. All patients gave their written informed consent [7].

The MARCH study design and methods were previously described in detail by Yang et al. [7] and Wang et al. [8]. Patients were randomized, after a 4-week run-in period, to receive up to $1500 \mathrm{mg}$ of sustained-release metformin hydrochloride $(500 \mathrm{mg}$ per tablet, Beijing Double Crane Pharma, Beijing, China) once daily or up to $300 \mathrm{mg} /$ day of acarbose (100 mg three times daily) (Bayer Healthcare, Beijing, China) with 24-week monotherapy and, as needed, a 24-week add-on therapy with insulin secretagogues. During the 4-week pre-randomization run-in period, dietary education was provided by trained dietitians and total daily calories were calculated based on standardized body weight and labor intensity. Patients were asked to perform 30 min of moderate exercise each day such as brisk walking, jogging, cycling, or swimming. As per the Chinese 2007 management guidelines for type 2 diabetes, add-on therapy with insulin secretagogues was initiated at week 24 in patients whose glycated haemoglobin $\left(\mathrm{HbA}_{1 \mathrm{c}}\right)$ levels were $>7 \%$, whose fasting plasma glucose (FPG) was $>7 \mathrm{mmol} / \mathrm{l}$, or whose postprandial glucose was $>10 \mathrm{mmol} / \mathrm{l}$ for three consecutive days.

\section{Measurements}

Baseline demographic and patient clinical characteristics were obtained including body weight, waist and hip circumference, body mass index (BMI), GLP-1 levels, glucose sensitivity, fasting serum insulin (FINS), total cholesterol, levels of low-density lipoproteins (LDL) and high-density lipoproteins $(\mathrm{HDL})$, and levels of triglycerides. Biochemical and anthropometric measurements were also performed at weeks 24 and 48. Area under the concentration curve (AUC) was used to evaluate serum levels of insulin, glucagon, and GLP-1.

\section{Statistical analysis}

In order to investigate the factors associated with weight change, the median change in weight from baseline to the 48-week follow-up endpoint was used; $2 \mathrm{~kg}$ was the median value of the reduction of body weight of all the subjects in the MARCH trial. Hence, a considerable weight change was defined as $\geq 2 \mathrm{~kg}$ over the 48-week follow-up period. Both baseline demographics and clinic variables and change from baseline at weeks 24 and 48 for certain clinical variables were assessed. Variables with normal distribution were presented as means and standard deviations (SD), and the independent $t$ test was used to compare variables between patient groups that had weight that changed by $<2 \mathrm{~kg}$ or $\geq 2 \mathrm{~kg}$. Variables without normal distribution were presented as medians and inter-quartile ranges (IQRs), and the Wilcoxon rank sum test was used to compare the differences between two weight groups. Univariate and multivariate logistic regression models were performed to evaluate factors associated with $\geq 2 \mathrm{~kg}$ reduction in weight. Factors found in the univariate analysis to be associated with decreased weight were included in the multivariate analysis. For multivariate analysis, baseline factors and the changes from baseline at week 24 and week 48 were separated into different models.

\section{Statistical analysis}

Tests were considered significant if the two-sided $p$-value was $<0.05$. Statistical analyses were performed using the SAS 9.2 statistics software (SAS Inc., Cary, NC, USA).

\section{Results}

\section{Baseline demographics and clinical characteristics}

Patients who were treated with acarbose and who lost $<2 \mathrm{~kg}$ or $\geq 2 \mathrm{~kg}$ of weight differed in baseline demographic and clinical characteristic (Table I). A greater percentage of patients who lost $\geq 2 \mathrm{~kg}$ compared with those who lost $<2 \mathrm{~kg}$ 
MARCH: factors associated with weight loss in patients with newly diagnosed type 2 diabetes treated with acarbose or metformin

Table I. Baseline characteristics according to weight decrease $<2 \mathrm{~kg}$ vs. $\geq 2 \mathrm{~kg}$ in subjects treated with acarbose

\begin{tabular}{|c|c|c|c|}
\hline Parameter & $\begin{array}{l}\text { Weight decreased } \\
<2 \mathrm{~kg}(N=145)\end{array}$ & $\begin{array}{l}\text { Weight decreased } \\
\geq 2 \mathrm{~kg}(N=192)\end{array}$ & $P$-value \\
\hline \multicolumn{4}{|l|}{ Demographic characteristics: } \\
\hline Gender: & & & $0.041^{*}$ \\
\hline Male & $96(47.52 \%)$ & $106(52.48 \%)$ & \\
\hline Female & $49(36.3 \%)$ & $86(63.7 \%)$ & \\
\hline Age [years] & $50(43-55)$ & $51(44-57.5)$ & 0.161 \\
\hline $\mathrm{BMI}\left[\mathrm{kg} / \mathrm{m}^{2}\right]$ & $25.24(23.44-26.58)$ & $26.23(24.63-28.08)$ & $0.001^{*}$ \\
\hline Waist circumference $[\mathrm{cm}]$ & $89.33 \pm 8.53$ & $89.63 \pm 8.07$ & 0.749 \\
\hline 1-day energy intake from carbohydrates [cal] & $220.25(185.61-268.7)$ & $215.05(166.65-267.18)$ & 0.23 \\
\hline 1-day energy intake from non-carbohydrates [cal] & $107.57(81.57-137.57)$ & $103.85(73.02-127.56)$ & 0.091 \\
\hline \multicolumn{4}{|l|}{ Clinical characteristics: } \\
\hline Systolic blood pressure [mm Hg] & $122(115-130)$ & $125(120-131.5)$ & 0.132 \\
\hline Diastolic blood pressure [mm Hg] & $78.63 \pm 8.14$ & $80.45 \pm 8.08$ & $0.043^{*}$ \\
\hline Total cholesterol [mmol/l] & $5.12(4.38-5.76)$ & $5.18(4.57-5.99)$ & 0.355 \\
\hline LDL cholesterol [mmol/l] & $3(2.51-3.55)$ & $3.1(2.5-3.57)$ & 0.722 \\
\hline HDL cholesterol [mmol/l] & $1.2(1-1.42)$ & $1.18(1.05-1.39)$ & 0.813 \\
\hline Triglycerides $[\mathrm{mmol} / \mathrm{l}]$ & $1.77(1.26-2.52)$ & $1.84(1.2-2.7)$ & 0.877 \\
\hline \multicolumn{4}{|l|}{ Glycemic characteristics: } \\
\hline \multicolumn{4}{|l|}{$\mathrm{HbA}_{1 \mathrm{c}}(\%):$} \\
\hline Baseline & $7.5(6.8-8.3)$ & $7.1(6.5-7.9)$ & $0.001^{\star}$ \\
\hline Difference between 24 weeks and baseline & $-1(-1.8--0.2)$ & $-1(-1.6--0.4)$ & 0.715 \\
\hline Difference between 48 weeks and baseline & $-0.8(-1.5--0.2)$ & $-1(-1.7--0.3)$ & 0.081 \\
\hline \multicolumn{4}{|l|}{ FPG [mmol/l]: } \\
\hline Baseline & $8.7(7.85-9.9)$ & $8.6(7.65-9.75)$ & 0.357 \\
\hline Difference between 24 weeks and baseline & $-1.65(-2.6--0.75)$ & $-1.8(-3.05--0.93)$ & $0.032^{*}$ \\
\hline Difference between 48 weeks and baseline & $-1.5(-2.25--0.55)$ & $-2.18(-3.1--1.13)$ & $<0.001^{*}$ \\
\hline \multicolumn{4}{|l|}{$2 \mathrm{~h}-\mathrm{PPG}[\mathrm{mmol} / \mathrm{l}]:$} \\
\hline Baseline & $12.74 \pm 2.78$ & $12.36 \pm 2.8$ & 0.22 \\
\hline Difference between 24 weeks and baseline & $-2.51 \pm 3.03$ & $-3.43 \pm 2.8$ & $0.004^{*}$ \\
\hline Difference between 48 weeks and baseline & $-2.31 \pm 3.2$ & $-3.59 \pm 3.05$ & $<0.001^{*}$ \\
\hline \multicolumn{4}{|l|}{ Insulin, glucagon, and GLP-1 characteristics: } \\
\hline \multicolumn{4}{|l|}{ HOMA-B: } \\
\hline Baseline & $47.1(29.16-74.3)$ & $48.19(28.86-76.2)$ & 0.996 \\
\hline Difference between 24 weeks and baseline & $4.33(-24.08-23.52)$ & $3.65(-19.55-29.58)$ & 0.493 \\
\hline Difference between 48 weeks and baseline & $-3.05(-24.35-19.85)$ & $0.05(-18.91-26.28)$ & 0.358 \\
\hline \multicolumn{4}{|l|}{ HOMA-IR: } \\
\hline Baseline & $3.79(2.49-6.06)$ & $3.84(2.44-6.13)$ & 0.864 \\
\hline Difference between 24 weeks and baseline & $-1.07(-3.02-0.75)$ & $-1.5(-3.72--0.15)$ & 0.069 \\
\hline Difference between 48 weeks and baseline & $-1.19(-3.5-0.28)$ & $-1.69(-4--0.39)$ & $0.028^{*}$ \\
\hline
\end{tabular}


Table I. Cont.

\begin{tabular}{|c|c|c|c|}
\hline Parameter & $\begin{array}{l}\text { Weight decreased } \\
<2 \mathrm{~kg}(N=145)\end{array}$ & $\begin{array}{l}\text { Weight decreased } \\
\geq 2 \mathrm{~kg}(N=192)\end{array}$ & $P$-value \\
\hline \multicolumn{4}{|l|}{ Early insulin secretion index: } \\
\hline Baseline & $2.33(0.8-4.23)$ & $2.62(1.21-4.8)$ & 0.096 \\
\hline Difference between 24 weeks and baseline & $1.04(-2.12-3.94)$ & $0.89(-2.52-3.77)$ & 0.495 \\
\hline Difference between 48 weeks and baseline & $0.39(-2.78-3.49)$ & $0.54(-2.52-4.21)$ & 0.889 \\
\hline \multicolumn{4}{|l|}{ Whole body insulin sensitivity index: } \\
\hline Baseline & $4.1(2.86-5.56)$ & $3.78(2.57-5.89)$ & 0.438 \\
\hline Difference between 24 weeks and baseline & $1.77(-0.14-4.53)$ & $3.33(0.81-6.48)$ & $0.001^{*}$ \\
\hline Difference between 48 weeks and baseline & $1.96(-0.09-4.01)$ & $3.94(1.33-7.11)$ & $<0.001^{*}$ \\
\hline \multicolumn{4}{|l|}{ AUC for serum insulin $[\mu \mid \mathrm{I} / \mathrm{ml} \times \mathrm{min}]$ : } \\
\hline Baseline & $4109.55(3014.4-5676.15)$ & $4653.3(3347.55-6527.7)$ & 0.092 \\
\hline Difference between 24 weeks and baseline & $-790.35(-1875-385.2)$ & $-1253.25(-2661-202.35)$ & 0.093 \\
\hline Difference between 48 weeks and baseline & $\begin{array}{c}-371.1 \\
(-1634.4-756.3)\end{array}$ & $\begin{array}{c}-1448.78 \\
(-3199.28--121.05)\end{array}$ & $<0.001^{*}$ \\
\hline \multicolumn{4}{|l|}{ AUC for glucagon $[\mathrm{pg} / \mathrm{ml} \times \mathrm{min}]$} \\
\hline Baseline & $\begin{array}{c}11873.85 \\
(9289.5-15032.25) \\
\end{array}$ & $\begin{array}{c}12447.75 \\
(10174.05-17038.5)\end{array}$ & 0.06 \\
\hline Difference between 24 weeks and baseline & $\begin{array}{c}-461.7 \\
(-4019.4-3575.55)\end{array}$ & $\begin{array}{c}-1209.45 \\
(-5413.2-3302.85)\end{array}$ & 0.211 \\
\hline Difference between 48 weeks and baseline & $\begin{array}{c}-1493.48 \\
(-5351.63-2853.23) \\
\end{array}$ & $\begin{array}{c}-2677.35 \\
(-6003.75-1195.5) \\
\end{array}$ & 0.072 \\
\hline \multicolumn{4}{|l|}{ AUC for plasma GLP-1 [pmol × min]: } \\
\hline Baseline & $\begin{array}{c}3032.33 \\
(1845.53-4829.4) \\
\end{array}$ & $\begin{array}{c}2490.23 \\
(1643.78-4181.78) \\
\end{array}$ & 0.05 \\
\hline Difference between 24 weeks and baseline & $897.15(-836.7-2640.9)$ & $1188.45(-300.3-2760.6)$ & 0.14 \\
\hline Difference between 48 weeks and baseline & $787.95(-723.15-2745.3)$ & $1443(-180-2620.05)$ & 0.247 \\
\hline
\end{tabular}

of weight over the course of the study were female $(63.7 \%$ vs. $36.3 \%$, respectively; $p=0.041)$, had higher baseline BMI $\left(26.23 \mathrm{~kg} / \mathrm{m}^{2}\right.$ vs. 25.24 $\left.\mathrm{kg} / \mathrm{m}^{2} ; p=0.001\right)$, and had lower baseline $\mathrm{HbA}_{1 \mathrm{c}}$ ( $p<0.001)$. Patients with $\geq 2 \mathrm{~kg}$ of weight reduction also had greater decreases from baseline in FPG and 2-hour postprandial glucose (2 h-PPG) (FPG at week $48 ;-2.18 \mathrm{mmol} / \mathrm{l}$ vs. $-1.5 \mathrm{mmol} / \mathrm{l}$; $p<0.001 ; 2$ h-PPG at week 48 : -3.59 vs. -2.31 ; $p<0.001)$ and greater increases from baseline in the whole body insulin sensitivity index at weeks 24 and 48 (week 24: 3.33 vs. 1.77, $p=0.001$; week 48: 3.94 vs., $1.96, p<0.001$ ). Patients with $\geq 2 \mathrm{~kg}$ weight loss also had greater decreases from baseline in HOMA-IR (-1.69 vs. $-1.19, p=0.028)$ and larger reductions from baseline in insulin AUC $(-1448.78 \mu \mathrm{lU} / \mathrm{ml} \times \mathrm{min}$ vs. $-371.1 \mu \mathrm{lU} / \mathrm{ml} \times \mathrm{min}$; $p<0.001)$ at week 48.

In patients treated with metformin, $\geq 2 \mathrm{~kg}$ weight loss compared with $<2 \mathrm{~kg}$ reduction in weight was associated with higher baseline
BMI $\left(26.08 \mathrm{~kg} / \mathrm{m}^{2}\right.$ vs. $25.21 \mathrm{~kg} / \mathrm{m}^{2}$, respectively; $p=0.002)$, lower baseline $\mathrm{HbA}_{1 \mathrm{c}}$ (7.3 vs. 7.7; $p=$ 0.003 ), lower baseline 2h-PPG (12.34 vs. 13.01; $p=0.04)$, and higher baseline HOMA-B (57.61 vs. $45.2 ; p=0.007$ ) (Table II). Metformin patients with $\geq 2 \mathrm{~kg}$ weight reduction also had greater decreases from baseline in HOMA-B (-3.02 vs. 6.35, $p=0.048)$ and HOMA-IR (-2.21 vs. -1.48 , $p=0.004)$, and a larger reduction from baseline in FPG (at week 48: -2.35 vs. $-1.73 ; p=0.033$ ) at weeks 48. Patients treated with metformin who lost $\geq 2 \mathrm{~kg}$ of weight also had greater increases from baseline in the whole body insulin sensitivity index at 24 and 48 weeks (week 24: 2.24 vs. 1.89, $p=0.047$; week 48: 2.98 vs. $1.8, p<0.001$ ).

\section{Acarbose treated patients}

Univariate analysis identified the following demographic and clinical characteristics as being associated with $a \geq 2 \mathrm{~kg}$ loss in weight: gender, 
MARCH: factors associated with weight loss in patients with newly diagnosed type 2 diabetes treated with acarbose or metformin

Table II. Baseline characteristics according to weight decrease $<2 \mathrm{~kg} \mathrm{vs.} \geq 2 \mathrm{~kg}$ in subjects treated with metformin

\begin{tabular}{|c|c|c|c|}
\hline Parameter & $\begin{array}{l}\text { Weight decreased }<2 \mathrm{~kg} \\
(N=144)\end{array}$ & $\begin{array}{l}\text { Weight decreased } \geq 2 \mathrm{~kg} \\
(N=183)\end{array}$ & $P$-value \\
\hline \multicolumn{4}{|l|}{ Demographic characteristics: } \\
\hline Gender: & & & 0.176 \\
\hline Male & $94(47 \%)$ & $106(53 \%)$ & \\
\hline Female & $50(39.37 \%)$ & 77 (60.63\%) & \\
\hline Age [years] & $49(41-56.5)$ & $52(44-57)$ & 0.099 \\
\hline $\mathrm{BMI}\left[\mathrm{kg} / \mathrm{m}^{2}\right]$ & $25.21(23.61-26.79)$ & $26.08(24.21-28.39)$ & $0.002^{*}$ \\
\hline Waist circumference $[\mathrm{cm}]$ & $89.3 \pm 8.33$ & $89.96 \pm 8.2$ & 0.473 \\
\hline 1-day energy intake from carbohydrates [cal] & $210.07(167.89-257.83)$ & $219.09(162.14-264.7)$ & 0.772 \\
\hline 1-day energy intake from non-carbohydrates [cal] & $105.57(75.79-137.59)$ & $99.15(74.65-130.44)$ & 0.384 \\
\hline \multicolumn{4}{|l|}{ Clinical characteristics: } \\
\hline Systolic blood pressure [mm Hg] & $120(118-130)$ & $125(120-135)$ & 0.098 \\
\hline Diastolic blood pressure [mm Hg] & $78.88 \pm 7.54$ & $81.33 \pm 7.1$ & $0.003^{*}$ \\
\hline Total cholesterol [mmol/l] & $5.12(4.31-5.93)$ & $5.13(4.43-5.88)$ & 0.96 \\
\hline LDL cholesterol [mmol/l] & $2.97(2.41-3.65)$ & $2.95(2.42-3.58)$ & 0.872 \\
\hline HDL cholesterol [mmol/l] & $1.18(1-1.36)$ & $1.18(1.03-1.38)$ & 0.893 \\
\hline Triglycerides [mmol/l] & $1.98(1.39-2.83)$ & $1.99(1.38-2.76)$ & 0.844 \\
\hline \multicolumn{4}{|l|}{ Glycemic characteristics: } \\
\hline \multicolumn{4}{|l|}{$\mathrm{HbA}_{1 \mathrm{c}}(\%):$} \\
\hline Baseline & $7.7(6.85-8.7)$ & $7.3(6.7-7.9)$ & $0.003^{*}$ \\
\hline Difference between 24 weeks and baseline & $-1.1(-2--0.5)$ & $-1.1(-1.7--0.5)$ & 0.521 \\
\hline Difference between 48 weeks and baseline & $-0.8(-1.9--0.2)$ & $-1(-1.7--0.5)$ & 0.424 \\
\hline \multicolumn{4}{|l|}{$\mathrm{FPG}[\mathrm{mmol} / \mathrm{ll}]$} \\
\hline Baseline & $8.75(7.88-9.98)$ & $8.65(7.95-9.65)$ & 0.627 \\
\hline Difference between 24 weeks and baseline & $-1.9(-2.7--1.2)$ & $-2.1(-3.05--1.3)$ & 0.213 \\
\hline Difference between 48 weeks and baseline & $-1.73(-2.88--0.98)$ & $-2.35(-3.25--1.15)$ & $0.033^{*}$ \\
\hline \multicolumn{4}{|l|}{ 2h-PPG: } \\
\hline Baseline & $13.01(10.74-15.1)$ & $12.26(10-14.63)$ & $0.04^{*}$ \\
\hline Difference between 24 weeks and baseline & $-2.55(-4.47-0.1)$ & $-2.75(-4.6--1)$ & 0.324 \\
\hline Difference between 48 weeks and baseline & $-1.91(-4.39-0.2)$ & $-2.58(-4.82--0.57)$ & 0.124 \\
\hline \multicolumn{4}{|l|}{ Insulin, glucagon, and GLP-1 characteristics: } \\
\hline \multicolumn{4}{|l|}{ HOMA-B: } \\
\hline Baseline & $45.2(27.42-68.19)$ & $57.61(35.67-79.82)$ & $0.007^{\star}$ \\
\hline Difference between 24 weeks and baseline & $10.77(-5.88-29.24)$ & $2.15(-19.35-31.78)$ & 0.120 \\
\hline Difference between 48 weeks and baseline & $6.35(-10.47-28.97)$ & $-3.02(-28.64-27.45)$ & $0.048^{*}$ \\
\hline \multicolumn{4}{|l|}{ HOMA-IR: } \\
\hline Baseline & $4.41(2.64-6.4)$ & $4.42(2.71-6.79)$ & 0.512 \\
\hline Difference between 24 weeks and baseline & $-1.46(-3.31--0.05)$ & $-1.99(-3.66--0.49)$ & 0.123 \\
\hline Difference between 48 weeks and baseline & $-1.48(-3.25-0.18)$ & $-2.21(-4.71--0.91)$ & $0.004^{*}$ \\
\hline
\end{tabular}


Table II. Cont.

\begin{tabular}{|c|c|c|c|}
\hline Parameter & $\begin{array}{l}\text { Weight decreased }<2 \mathrm{~kg} \\
\quad(N=144)\end{array}$ & $\begin{array}{l}\text { Weight decreased } \geq 2 \mathrm{~kg} \\
\quad(N=183)\end{array}$ & $P$-value \\
\hline \multicolumn{4}{|l|}{ Early insulin secretion index: } \\
\hline Baseline & $2.56(1-3.96)$ & $2.52(1.1-4.64)$ & 0.732 \\
\hline Difference between 24 weeks and baseline & $0.69(-2.37-3.25)$ & $1.87(-1.19-4.66)$ & 0.059 \\
\hline Difference between 48 weeks and baseline & $0.41(-2.36-2.37)$ & $1.22(-1.08-3.26)$ & $0.017^{*}$ \\
\hline \multicolumn{4}{|l|}{ Whole body insulin sensitivity index: } \\
\hline Baseline & $3.79(2.63-5.53)$ & $3.34(2.47-5.41)$ & 0.406 \\
\hline Difference between 24 weeks and baseline & $1.89(0.16-4.89)$ & $2.24(0.95-5.7)$ & $0.047^{\star}$ \\
\hline Difference between 48 weeks and baseline & $1.8(-0.12-3.79)$ & $2.98(1.24-6.25)$ & $<0.001^{*}$ \\
\hline \multicolumn{4}{|l|}{ AUC for serum insulin $[\mu \mathrm{IU} / \mathrm{ml} \times \mathrm{min}]$} \\
\hline Baseline & $\begin{array}{c}4154.48 \\
(2994.75-6009)\end{array}$ & $\begin{array}{c}4708.95 \\
(3286.65-6520.65)\end{array}$ & 0.171 \\
\hline Difference between 24 weeks and baseline & $\begin{array}{c}-106.2 \\
(-1377.45-1030.65)\end{array}$ & $\begin{array}{c}-451.95 \\
(-2229.45-696.45)\end{array}$ & 0.080 \\
\hline Difference between 48 weeks and baseline & $\begin{array}{c}-499.2 \\
(-1588.2-1320.45)\end{array}$ & $\begin{array}{c}-584.85 \\
(-2296.35-696)\end{array}$ & 0.110 \\
\hline \multicolumn{4}{|l|}{ AUC for glucagon $[\mathrm{pg} / \mathrm{ml} \times \mathrm{min}]$ : } \\
\hline Baseline & $\begin{array}{c}12689.03 \\
(9594.15-17729.25)\end{array}$ & $\begin{array}{c}12507.6 \\
(9700.95-15334.5)\end{array}$ & 0.201 \\
\hline Difference between 24 weeks and baseline & $\begin{array}{c}35.03 \\
(-4197.15-5356.65)\end{array}$ & $\begin{array}{c}-350.1 \\
(-4138.05-3260.7)\end{array}$ & 0.524 \\
\hline Difference between 48 weeks and baseline & $\begin{array}{c}-1823.7 \\
(-5935.5-2001.9)\end{array}$ & $\begin{array}{c}-2461.65 \\
(-5740.8-1197.6) \\
\end{array}$ & 0.390 \\
\hline \multicolumn{4}{|l|}{ AUC for plasma GLP-1 [pmol x min]: } \\
\hline Baseline & $\begin{array}{c}2878.2 \\
(1748.1-4873.2)\end{array}$ & $\begin{array}{c}2773.05 \\
(1702.2-4896.45)\end{array}$ & 0.896 \\
\hline Difference between 24 weeks and baseline & $\begin{array}{c}1131 \\
(-143.4-2738.85)\end{array}$ & $\begin{array}{c}1115.4 \\
(-877.05-2763.45)\end{array}$ & 0.553 \\
\hline Difference between 48 weeks and baseline & $\begin{array}{c}1448.55 \\
(-550.58-2825.48)\end{array}$ & $\begin{array}{c}1252.05 \\
(-411.45-3047.1)\end{array}$ & 0.928 \\
\hline
\end{tabular}

FPG - fasting plasma glucose, $2 \mathrm{~h}$-PPG - $2 \mathrm{~h}$ postprandial glucose. ${ }^{*} P<0.05$, significant differences between weight decreased $<2 \mathrm{~kg}$ vs. $\geq 2 \mathrm{~kg}$.

baseline $\mathrm{HbA}_{1 \mathrm{c}}$ levels, and glucagon and GLP-1 AUCs ( $p$-values < 0.05) (Table III). Change from baseline in FPG and PPG levels and the whole body insulin sensitivity index at weeks 24 and 48 , and in HOMA-IR and in insulin AUC at week 48 were also associated with $\mathrm{a} \geq 2 \mathrm{~kg}$ weight reduction ( $p$-values $\leq 0.038$ ).

The factors significantly associated with loss of $\geq 2 \mathrm{~kg}$ of weight detected by univariate analyses were included in multivariate analyses. For baseline factors, multivariate analysis revealed that gender, $\mathrm{HbA}_{1 \mathrm{c}}$ levels and glucagon AUC were associated with $\geq 2 \mathrm{~kg}$ of weight loss (Table IV). $A$ reduction in weight of $\geq 2 \mathrm{~kg}$ was less likely for males than females $(\mathrm{OR}=0.58, p=0.025)$ and in patients with higher baseline levels of $\mathrm{HbA}_{1 \mathrm{c}}$ $(\mathrm{OR}=0.77, p=0.012)$. Reduction in $\geq 2 \mathrm{~kg}$ of weight was positively associated with higher baseline glucagon AUC (OR $=1.00006, p=0.010)$. No significant associations were found for reduction in weight of $\geq 2 \mathrm{~kg}$ and any assessed factors at week 24 (all $p>0.05$ ). Smaller increases from baseline at week 48 in FPG $(O R=0.65, p=0.0002)$ and insulin AUC (OR $=0.9998, p=0.008)$ were negatively associated with $\geq 2 \mathrm{~kg}$ reduction in weight.

\section{Metformin treatment}

Univariate analysis found that in patients treated with metformin the demographic and clinical characteristics that influenced a reduction of weight of $\geq 2 \mathrm{~kg}$ were baseline $\mathrm{HbA}_{1 \mathrm{c}}$ levels and PPG levels, changes at 48 weeks in FPG, whole body insulin sensitivity index, and insulin AUC (all $p$-values < 0.05) (Table V). Since no changes from baseline at week 24 were significantly 
Table III. Univariate analyses to detect baseline factors associated with weight change of $\geq 2 \mathrm{~kg}$ in subjects treated with acarbose

\begin{tabular}{|c|c|c|}
\hline \multirow[t]{2}{*}{ Parameter } & \multicolumn{2}{|c|}{ Univariate analysis } \\
\hline & OR $(95 \% \mathrm{Cl})$ & $P$-value \\
\hline Gender (ref = female) & $0.63(0.4-0.98)$ & $0.042^{*}$ \\
\hline Age [years] & $1.02(0.99-1.04)$ & 0.151 \\
\hline Waist circumference $[\mathrm{cm}]$ & $1.004(0.98-1.03)$ & 0.748 \\
\hline 1-day energy intake from carbohydrates [cal] & $0.998(0.996-1.001)$ & 0.258 \\
\hline 1-day energy intake from non-carbohydrates [cal] & $0.997(0.992-1.001)$ & 0.146 \\
\hline Total cholesterol $[\mathrm{mmol} / \mathrm{l}]$ & $1.1(0.9-1.35)$ & 0.334 \\
\hline LDL cholesterol [mmol/l] & $1.02(0.8-1.3)$ & 0.895 \\
\hline HDL cholesterol [mmol/l] & $0.97(0.45-2.08)$ & 0.931 \\
\hline Triglycerides [mmol/l] & $1.04(0.94-1.14)$ & 0.475 \\
\hline \multicolumn{3}{|l|}{$\mathrm{HbA}_{1 \mathrm{c}}(\%):$} \\
\hline Baseline & $0.76(0.63-0.92)$ & $0.004^{*}$ \\
\hline Difference between 24 weeks and baseline & $0.99(0.83-1.18)$ & 0.934 \\
\hline Difference between 48 weeks and baseline & $0.9(0.75-1.07)$ & 0.229 \\
\hline \multicolumn{3}{|l|}{ FPG $[\mathrm{mmol} / \mathrm{l}]:$} \\
\hline Baseline & $0.92(0.76-1.1)$ & 0.349 \\
\hline Difference between 24 weeks and baseline & $0.84(0.72-0.99)$ & $0.034^{*}$ \\
\hline Difference between 48 weeks and baseline & $0.7(0.59-0.82)$ & $<0.001^{*}$ \\
\hline \multicolumn{3}{|l|}{ 2h-PPG [mmol/l]: } \\
\hline Baseline & $0.95(0.88-1.03)$ & 0.22 \\
\hline Difference between 24 weeks and baseline & $0.9(0.83-0.97)$ & $0.005^{*}$ \\
\hline Difference between 48 weeks and baseline & $0.88(0.82-0.94)$ & $<0.001^{*}$ \\
\hline \multicolumn{3}{|l|}{ HOMA-B: } \\
\hline Baseline & $1.001(0.9976-1.004)$ & 0.579 \\
\hline Difference between 24 weeks and baseline & $0.9998(0.998-1.001)$ & 0.809 \\
\hline Difference between 48 weeks and baseline & $1.001(0.999-1.003)$ & 0.514 \\
\hline \multicolumn{3}{|l|}{ HOMA-IR: } \\
\hline Baseline & $1.01(0.95-1.07)$ & 0.71 \\
\hline Difference between 24 weeks and baseline & $0.95(0.89-1.01)$ & 0.099 \\
\hline Difference between 48 weeks and baseline & $0.94(0.88-0.996)$ & $0.038^{*}$ \\
\hline \multicolumn{3}{|l|}{ Early insulin secretion index: } \\
\hline Baseline & $1.02(0.99-1.06)$ & 0.134 \\
\hline Difference between 24 weeks and baseline & $0.997(0.98-1.01)$ & 0.666 \\
\hline Difference between 48 weeks and baseline & $1.002(0.99-1.01)$ & 0.654 \\
\hline \multicolumn{3}{|l|}{ Whole body insulin sensitivity index: } \\
\hline Baseline & $0.999(0.92-1.08)$ & 0.989 \\
\hline Difference between 24 weeks and baseline & $1.08(1.02-1.14)$ & $0.009^{*}$ \\
\hline Difference between 48 weeks and baseline & $1.15(1.08-1.22)$ & $<0.001^{*}$ \\
\hline
\end{tabular}


Table III. Cont.

\begin{tabular}{|c|c|c|}
\hline \multirow[t]{2}{*}{ Parameter } & \multicolumn{2}{|c|}{ Univariate analysis } \\
\hline & OR $(95 \% \mathrm{Cl})$ & $P$-value \\
\hline \multicolumn{3}{|l|}{ AUC for serum insulin at baseline $[\mu \mathrm{IU} / \mathrm{ml} \times \mathrm{min}]$ : } \\
\hline Baseline & $1.0001(0.99997-1.0002)$ & 0.163 \\
\hline Difference between 24 weeks and baseline & $0.9999(0.9998-1.00002)$ & 0.12 \\
\hline Difference between 48 weeks and baseline & $0.9998(0.9997-0.9999)$ & $<0.001^{*}$ \\
\hline \multicolumn{3}{|l|}{ AUC for glucagon $[\mathrm{pg} / \mathrm{ml} \times \mathrm{min}]$ : } \\
\hline Baseline & $1.00005(1.00001-1.00009)$ & $0.019^{*}$ \\
\hline Difference between 24 weeks and baseline & $0.99997(0.9999-1.00001)$ & 0.115 \\
\hline Difference between 48 weeks and baseline & $0.99996(0.9999-0.999999)$ & $0.047^{\star}$ \\
\hline \multicolumn{3}{|l|}{ AUC for plasma GLP-1 [pmol x min]: } \\
\hline Baseline & $0.9999(0.9998-0.999995)$ & $0.041^{*}$ \\
\hline Difference between 24 weeks and baseline & $1.0001(0.99999-1.0002)$ & 0.103 \\
\hline Difference between 48 weeks and baseline & $1.00005(0.99995-1.0001)$ & 0.326 \\
\hline
\end{tabular}

$F P G$ - fasting plasma glucose, $2 h-P P G-2 h$ postprandial glucose. ${ }^{*} P<0.05$, significantly associated with considerable weight change.

Table IV. Multivariate analyses to detect factors associated with weight change of $\geq 2 \mathrm{~kg}$ in subjects treated with acarbose

\begin{tabular}{|c|c|c|}
\hline \multirow[t]{2}{*}{ Variable } & \multicolumn{2}{|c|}{ Multivariate analysis } \\
\hline & OR $(95 \% \mathrm{Cl})$ & $P$-value \\
\hline \multicolumn{3}{|l|}{ For baseline variables: } \\
\hline Gender (ref = female) & $0.58(0.37-0.93)$ & $0.025^{*}$ \\
\hline $\mathrm{HbA}_{1 \mathrm{c}}(\%)$ & $0.77(0.63-0.95)$ & $0.012^{*}$ \\
\hline AUC for glucagon $[\mathrm{pg} / \mathrm{ml} \times \mathrm{min}]$ & $1.00006(1.00001-1.0001)$ & $0.010^{*}$ \\
\hline AUC for plasma GLP-1 $[\mathrm{pmol} \times \mathrm{min}]$ & $0.9999(0.9998-1.00004)$ & 0.230 \\
\hline \multicolumn{3}{|l|}{ For differences at 24 weeks: } \\
\hline$\Delta \mathrm{FPG}$ between 24 weeks and baseline & $0.89(0.75-1.05)$ & 0.170 \\
\hline$\triangle P P G$ between 24 weeks and baseline & $0.92(0.84-1.01)$ & 0.072 \\
\hline$\Delta$ Whole body insulin sensitivity index between 24 weeks and baseline & $1.06(0.999-1.12)$ & 0.056 \\
\hline \multicolumn{3}{|l|}{ For differences at 48 weeks: } \\
\hline$\Delta \mathrm{FPG}$ between 48 weeks and baseline & $0.67(0.54-0.83)$ & $0.0002^{*}$ \\
\hline$\triangle P P G$ between 48 weeks and baseline & $0.95(0.86-1.05)$ & 0.323 \\
\hline$\triangle \mathrm{HOMA}-\mathrm{IR}$ between 48 weeks and baseline & $1.01(0.93-1.11)$ & 0.754 \\
\hline$\Delta$ Whole body insulin sensitivity index between 48 weeks and baseline & $1.06(0.98-1.14)$ & 0.151 \\
\hline$\triangle A U C$ for serum insulin between 48 weeks and baseline & $0.9998(0.9997-0.99995)$ & $0.008^{*}$ \\
\hline$\triangle A U C$ for glucagon between 48 weeks and baseline & $0.99998(0.9999-1.00002)$ & 0.250 \\
\hline
\end{tabular}

Baseline factors and the changes from baseline at week 24 and week 48 were separated into different models. FPG - fasting plasma glucose, $2 \mathrm{~h}-P P G-2 \mathrm{~h}$ postprandial glucose. ${ }^{*} P<0.05$, significantly associated with considerable weight change.

associated with a reduction of weight of $\geq 2 \mathrm{~kg}$ in univariate analyses, no multivariate analysis was performed for changes at week 24. At week 48, multivariate analysis revealed that baseline $\mathrm{HbA}_{1 c}$ levels were negatively associated with $\geq 2 \mathrm{~kg}$ weight reduction $(\mathrm{OR}=0.80, p=0.036)$. In addition, the analysis revealed that increase from baseline in the whole body insulin sensitivity index at week 48 increased the likelihood of weight reduction $(\mathrm{OR}=1.10, p=0.014)$ (Table VI). 
Table V. Univariate analyses to detect the baseline factors associated with weight change of $\geq 2 \mathrm{~kg}$ in subjects treated with metformin

\begin{tabular}{|c|c|c|}
\hline \multirow[t]{2}{*}{ Variable } & \multicolumn{2}{|c|}{ Univariate analysis } \\
\hline & OR $(95 \% \mathrm{Cl})$ & $P$-value \\
\hline Gender (ref = female) & $0.73(0.47-1.15)$ & 0.176 \\
\hline Age [years] & $1.02(0.995-1.04)$ & 0.127 \\
\hline Waist circumference $[\mathrm{cm}]$ & $1.01(0.98-1.04)$ & 0.472 \\
\hline 1-day energy intake from carbohydrates [cal] & $1.0004(0.9974-1.0034)$ & 0.794 \\
\hline 1-day energy intake from non-carbohydrates [cal] & $0.997(0.99-1.002)$ & 0.269 \\
\hline Total cholesterol [mmol/l] & $1.01(0.84-1.23)$ & 0.908 \\
\hline LDL cholesterol [mmol/l] & $0.96(0.76-1.21)$ & 0.717 \\
\hline HDL cholesterol [mmol/l] & $1.14(0.57-2.27)$ & 0.716 \\
\hline Triglycerides $[\mathrm{mmol} / \mathrm{l}]$ & $0.99(0.89-1.11)$ & 0.887 \\
\hline \multicolumn{3}{|l|}{$\mathrm{HbA}_{1 c}(\%):$} \\
\hline Baseline & $0.77(0.64-0.92)$ & $0.005^{\star}$ \\
\hline Difference between 24 weeks and baseline & $1.08(0.9-1.31)$ & 0.396 \\
\hline Difference between 48 weeks and baseline & $0.96(0.81-1.14)$ & 0.666 \\
\hline \multicolumn{3}{|l|}{ FPG $[\mathrm{mmol} / \mathrm{l}]:$} \\
\hline Baseline & $0.94(0.77-1.14)$ & 0.522 \\
\hline Difference between 24 weeks and baseline & $0.91(0.77-1.08)$ & 0.284 \\
\hline Difference between 48 weeks and baseline & $0.83(0.72-0.97)$ & $0.016^{*}$ \\
\hline \multicolumn{3}{|l|}{ 2h-PPG [mmol/l]: } \\
\hline Baseline & $0.93(0.86-0.996)$ & $0.039^{\star}$ \\
\hline Difference between 24 weeks and baseline & $0.94(0.88-1.01)$ & 0.113 \\
\hline Difference between 48 weeks and baseline & $0.94(0.88-1.004)$ & 0.063 \\
\hline \multicolumn{3}{|l|}{ HOMA-B: } \\
\hline Baseline & $1.006(0.9997-1.012)$ & 0.061 \\
\hline Difference between 24 weeks and baseline & $0.999(0.99-1.003)$ & 0.531 \\
\hline Difference between 48 weeks and baseline & $0.999(0.997-1.002)$ & 0.678 \\
\hline \multicolumn{3}{|l|}{ HOMA-IR: } \\
\hline Baseline & $0.999(0.94-1.06)$ & 0.965 \\
\hline Difference between 24 weeks and baseline & $0.99(0.93-1.05)$ & 0.740 \\
\hline Difference between 48 weeks and baseline & $0.97(0.92-1.02)$ & 0.193 \\
\hline \multicolumn{3}{|l|}{ Early insulin secretion index: } \\
\hline Baseline & $0.99(0.97-1.01)$ & 0.322 \\
\hline Difference between 24 weeks and baseline & $1.01(0.99-1.02)$ & 0.502 \\
\hline Difference between 48 weeks and baseline & $1.01(0.99-1.04)$ & 0.166 \\
\hline \multicolumn{3}{|l|}{ Whole body insulin sensitivity index: } \\
\hline Baseline & $0.97(0.9-1.06)$ & 0.49 \\
\hline Difference between 24 weeks and baseline & $1.05(0.995-1.12)$ & 0.075 \\
\hline Difference between 48 weeks and baseline & $1.12(1.05-1.18)$ & $<0.001^{*}$ \\
\hline
\end{tabular}


Table V. Cont.

\begin{tabular}{|c|c|c|}
\hline \multirow[t]{2}{*}{ Variable } & \multicolumn{2}{|c|}{ Univariate analysis } \\
\hline & OR $(95 \% \mathrm{Cl})$ & $P$-value \\
\hline \multicolumn{3}{|l|}{ AUC for serum insulin $[\mu \mathrm{IU} / \mathrm{ml} \times \mathrm{min}]$ : } \\
\hline Baseline & $1.0001(0.99998-1.0002)$ & 0.138 \\
\hline Difference between 24 weeks and baseline & $0.9999(0.9998-1.000001)$ & 0.052 \\
\hline Difference between 48 weeks and baseline & $0.9999(0.9998-0.9999998)$ & $0.0497^{*}$ \\
\hline \multicolumn{3}{|l|}{ AUC for glucagon $[\mathrm{pg} / \mathrm{ml} \times \mathrm{min}]$ : } \\
\hline Baseline & $0.99996(0.99992-1.000002)$ & 0.062 \\
\hline Difference between 24 weeks and baseline & $0.99998(0.9999-1.00002)$ & 0.406 \\
\hline Difference between 48 weeks and baseline & $0.99998(0.9999-1.00002)$ & 0.382 \\
\hline \multicolumn{3}{|l|}{ AUC for plasma GLP-1 [pmol x min]: } \\
\hline Baseline & $1.00002(0.99991-1.0001)$ & 0.779 \\
\hline Difference between 24 weeks and baseline & $0.99997(0.9999-1.0001)$ & 0.528 \\
\hline Difference between 48 weeks and baseline & $0.99999(0.9999-1.0001)$ & 0.755 \\
\hline
\end{tabular}

$F P G$ - fasting plasma glucose, $2 h-P P G-2 h$ postprandial glucose; ${ }^{*} p<0.05$, significantly associated with considerable weight change.

Table VI. Multivariate analyses to detect the factors associated with weight change of $\geq 2 \mathrm{~kg}$ in subjects treated with metformin

\begin{tabular}{|c|c|c|}
\hline \multirow[t]{2}{*}{ Variable } & \multicolumn{2}{|c|}{ Multivariate analysis } \\
\hline & OR $(95 \% \mathrm{Cl})$ & $P$-value \\
\hline \multicolumn{3}{|l|}{ For baseline variables: } \\
\hline $\mathrm{HbA}_{1 \mathrm{c}}(\%)$ & $0.80(0.65-0.99)$ & $0.036^{*}$ \\
\hline $2 \mathrm{~h}-\mathrm{PPG}[\mathrm{mmol} / \mathrm{l}]$ & $0.96(0.89-1.04)$ & 0.348 \\
\hline \multicolumn{3}{|l|}{ For differences at 48 weeks: } \\
\hline$\Delta$ FPG between 48 weeks and baseline & $0.90(0.77-1.06)$ & 0.214 \\
\hline$\Delta$ Whole body insulin sensitivity index between 48 weeks and baseline & $1.10(1.02-1.18)$ & $0.014^{*}$ \\
\hline$\triangle A U C$ for serum insulin between 48 weeks and baseline & $0.99999(0.9999-1.0001)$ & 0.909 \\
\hline
\end{tabular}

\section{Discussion}

In this sub-analysis of the MARCH trial, we evaluated what demographic and clinical factors were associated with reduction in weight at the end of the 48-week follow-up period. We assessed the effect of baseline values of different parameters on weight loss in patients treated with acarbose or metformin. Multivariate analysis indicated that in patients treated with acarbose, males were less likely than females to lose weight. In addition, higher baseline $\mathrm{HbA}_{1 \mathrm{c}}$ levels and smaller decreases from baseline in FPG levels after 48 weeks of treatment were negatively associated with weight reduction, and higher baseline glucagon AUC was positively associated with weight loss. In patients treated with metformin, lower decreases in FPG levels from baseline after 48 weeks of treatment were negatively associated with weight reduction. In addition, greater changes from baseline in whole body insulin sensitivity after 48 weeks of treatment increased the likelihood of weight reduction in metformin patients.

Acarbose and metformin are associated with weight loss in type 2 diabetes, although the exact mechanisms are not entirely clear [9]. Acarbose appears to mediate its weight loss effect through promoting GLP-1 levels [4]. Metformin is thought to reduce weight via regulatory pathways in the brain, and also via adipose and gut-derived signals $[5,9]$. Similar to acarbose, metformin may also reduce hunger and alter carbohydrate absorption by raising GLP-1 levels [10, 11]. We saw little effect of GLP-1 levels on weight loss in our analysis for both acarbose and metformin. These findings may be 
limited by the sample size. It is possible that larger sample sizes may have had greater sensitivity for detecting the effect of changes in GLP-1 levels on weight. Additional studies designed to evaluate the relationship of GLP-1 and weight reduction in type 2 diabetes patients treated with metformin or acarbose are necessary.

We found that baseline glucagon AUC is significantly associated with weight loss in patients treated with acarbose but not in those treated with metformin. Previous studies have found that GLP-1, whose levels are affected by acarbose therapy, can affect glucagon levels [4, 11-18]. However, our findings did not reveal an association of GLP-1 with weight reduction, possibly suggesting that the effect of acarbose on glucagon levels can act independently of GLP-1 to influence weight. We also found that change in the whole body insulin sensitivity index from baseline after 48 weeks of metformin but not acarbose therapy was associated with weight loss. The difference between the two drugs with regard to the importance of insulin and glucagon control and weight loss may reflect the complexity of glycemic control and the insulin-glucagon interaction. Additional studies are necessary to further understand how the two therapies act to influence weight.

Our findings suggest that subjects with better glycemic control with metformin or acarbose treatment were more likely to lose weight. These findings are consistent with prior studies that found an association of glycemic control with weight loss [16-19]. The study of Bluher et al. specifically evaluated the hypothesis that the degree of weight change with the dipeptidyl peptidase-4 (DPP-4) inhibitor vildagliptin was dependent on the level of baseline glycemic control [18]. Using linear regression analysis, they identified an FPG level $(14.6 \mathrm{mmol} / \mathrm{l})$ in which there was no change in weight. FPG levels below this neutral point resulted in weight loss and above this point resulted in weight gain. They also found a similar relationship between baseline $\mathrm{HbA}_{1 \mathrm{c}}$ levels and weight change. The authors concluded that treatment results in weight loss when glucose or $\mathrm{HbA}_{1 c}$ levels are below a renal threshold. Due to the complexity of the regulatory pathways involved in controlling caloric intake, fat metabolism, insulin resistance, etc. [20, 21], the exact role of different factors related to glycemic control in influencing weight is unclear. It is possible that our findings are more reflective of the importance of glucose homoeostasis for other bodily processes, including weight, and not a specific role for any given factor.

The findings and interpretation of this study are limited by the fact that this was a secondary analysis of the MARCH study. In addition, we only eval- uated a subset of baseline parameters and clinical factors associated with type 2 diabetes using multivariate analysis. It is possible that other parameters/factors may also influence weight loss. As this was a secondary analysis of the MARCH study, we were unable to assess other variables, such as exercise, diet, etc., which may have impacted weight loss. A study in rats found that weight loss associated with metformin or sitagliptin, and the type of diet (high fat, high protein, or mixed diet), influenced the effect of drugs on triglyceride and postprandial blood glucose levels [22]. Also, it is unclear whether lifestyle changes impacted the findings as they were not factored into body weight determination. Although we cannot actually determine to what extent any patient changes in lifestyle measures during or after the 4-week education/preparation period influenced weight loss, we know that no significant changes were noted in patients' weight during the 4-week run-in period before the study began, when measured by treatment group [19]. The MARCH study was performed using solely Chinese patients. Therefore, it is not clear how the findings relate to other ethnic groups and geographical regions. In addition, the $\mathrm{BMI}$ in the patient population in the $\mathrm{MARCH}$ study ranged from $19 \mathrm{~kg} / \mathrm{m}^{2}$ to $30 \mathrm{~kg} / \mathrm{m}^{2}$; hence, our population did not include obese patients, and it is unclear whether our findings would be similar in an obese population of patients.

In conclusion, this study found that for both acarbose and metformin, the levels of FPG significantly impacted weight loss. In addition, baseline AUC for glucagon in patients treated with acarbose and change from baseline in whole body insulin sensitivity after 48 weeks of therapy in metformin-treated patients were important factors for weight reduction.

\section{Conflict of interest}

The authors declare no conflict of interest.

\section{References}

1. Heppner KM, Perez-Tilve D. GLP-1 based therapeutics: simultaneously combating T2DM and obesity. Front Neurosci 2015; 9: 92.

2. Look ARG, Wing RR, Bolin P, et al. Cardiovascular effects of intensive lifestyle intervention in type 2 diabetes. N Engl J Med 2013; 369: 145-54.

3. Inzucchi SE, Bergenstal RM, Buse JB, et al. Management of hyperglycemia in type 2 diabetes: a patient-centered approach: position statement of the American Diabetes Association (ADA) and the European Association for the Study of Diabetes (EASD). Diabetes Care 2012; 35: 1364-79.

4. McCarty MF, DiNicolantonio JJ. Acarbose, lente carbohydrate, and prebiotics promote metabolic health and longevity by stimulating intestinal production of GLP-1. Open Heart 2015; 2: e000205. 
5. Malin SK, Kashyap SR. Effects of metformin on weight loss: potential mechanisms. Curr Opin Endocrinol Diabetes Obes 2014; 21: 323-9.

6. Meneghini LF, Orozco-Beltran D, Khunti K, et al. Weight beneficial treatments for type 2 diabetes. J Clin Endocrinol Metab 2011; 96: 3337-53.

7. Yang W, Liu J, Shan Z, et al. Acarbose compared with metformin as initial therapy in patients with newly diagnosed type 2 diabetes: an open-label, non-inferiority randomised trial. Lancet Diabetes Endocrinol 2014; 2: 46-55.

8. Wang G, Liu J, Yang N, et al. MARCH2: comparative assessment of therapeutic effects of acarbose and metformin in newly diagnosed type 2 diabetes patients. PLoS One 2014; 9: e105698.

9. Mitri J, Hamdy O. Diabetes medications and body weight. Expert Opin Drug Saf 2009; 8: 573-84.

10. Mulherin AJ, Oh AH, Kim H, Grieco A, Lauffer LM, Brubaker PL. Mechanisms underlying metformin-induced secretion of glucagon-like peptide-1 from the intestinal L cell. Endocrinology 2011; 152: 4610-9.

11. Lindsay JR, Duffy NA, McKillop AM, et al. Inhibition of dipeptidyl peptidase IV activity by oral metformin in type 2 diabetes. Diabet Med 2005; 22: 654-7.

12. Baggio LL, Drucker DJ. Biology of incretins: GLP-1 and GIP. Gastroenterology 2007; 132: 2131-57.

13. Holst JJ, Vilsboll T, Deacon CF. The incretin system and its role in type 2 diabetes mellitus. Mol Cell Endocrinol 2009; 297: 127-36.

14. Scrocchi LA, Brown TJ, MaClusky N, et al. Glucose intolerance but normal satiety in mice with a null mutation in the glucagon-like peptide 1 receptor gene. Nat Med 1996; 2: 1254-8.

15. Kreymann B, Williams G, Ghatei MA, Bloom SR. Glucagon-like peptide-1 7-36: a physiological incretin in man. Lancet 1987; 2: 1300-4.

16. McAdam-Marx C, Bellows BK, Unni S, et al. Determinants of glycaemic control in a practice setting: the role of weight loss and treatment adherence (The DELTA Study). Int J Clin Pract 2014; 68: 1309-17.

17. Nguyen KT, Billington CJ, Vella A, et al. Preserved insulin secretory capacity and weight loss are the predominant predictors of glycemic control in patients with type 2 diabetes randomized to Roux-en-Y gastric bypass. Diabetes 2015; 64: 3104-10.

18. Bluher M, Schweizer A, Bader G, Foley JE. Changes in body weight after 24 weeks of vildagliptin therapy as a function of fasting glucose levels in patients with type 2 diabetes. Vasc Health Risk Manag 2014; 10: 661-4.

19. Wang N, Zhang JP, Xing XY, et al. Associations between changes in glucagon-like peptide- 1 and bodyweight reduction in patients receiving acarbose or metformin treatment. J Diabetes 2017; 9: 728-37.

20. Papaetis GS, Papakyriakou P, Panagiotou TN. Central obesity, type 2 diabetes and insulin: exploring a pathway full of thorns. Arch Med Sci 2015; 11: 463-82.

21. Ghomari-Boukhatem H, Bouchouicha A, Mekki K. Blood pressure, dyslipidemia and inflammatory factors are related to body mass index in scholar adolescents. Arch Med Sci 2017; 13: 46-52.

22. Yang J, Ba T, Chen L, et al. Effects of metformin and sitagliptin on glycolipid metabolism in type 2 diabetic rats on different diets. Arch Med Sci 2016; 12: 233-42. 